\title{
Uniting to Advance Diversity, Equity, and Inclusion in a Pandemic and Post-Pandemic World
}

Non peer-reviewed preprint for publication with the Blog of the European Association of Geochemistry January 2021. This piece is an extended feature.

\section{By Amy J. V. Riches ${ }^{1,2^{\star}}$, Olivier Pourret ${ }^{3}$, and Susan Little ${ }^{4}$}

${ }^{1}$ Visiting Honorary Fellow, University of Edinburgh, School of Geoscience, Grant Institute, Edinburgh, EH9 3FE, UK ${ }^{2}$ SETI Institute, 189 Bernardo Ave, Suite 200, Mountain View, CA 94043, United States ${ }^{3}$ UniLaSalle, AGHYLE, Beauvais, SFR Condorcet FR CNRS 3417, France.

${ }^{4}$ University College London, Department of Earth Sciences, Gower Place, London, WC1E 6BS, UK *Corresponding author: ajvriches@gmail.com and contact dei@eag.eu.com 


\section{Uniting to Advance Diversity, Equity, and Inclusion in a Pandemic and Post-Pandemic World}

\section{THE WORK}

There are significant numbers of highly-educated, well-trained, talented and hardworking people in geochemistry and wider Science, Technology, Engineering, and Maths (STEM) fields around the globe, and over recent decades we have witnessed substantial increases in the annual number of new PhDs. To best serve excellence among all and within every sector, diverse careers must be recognised and valued, and system models must be substantially reformed to better equip people for a variety of career paths / destinations

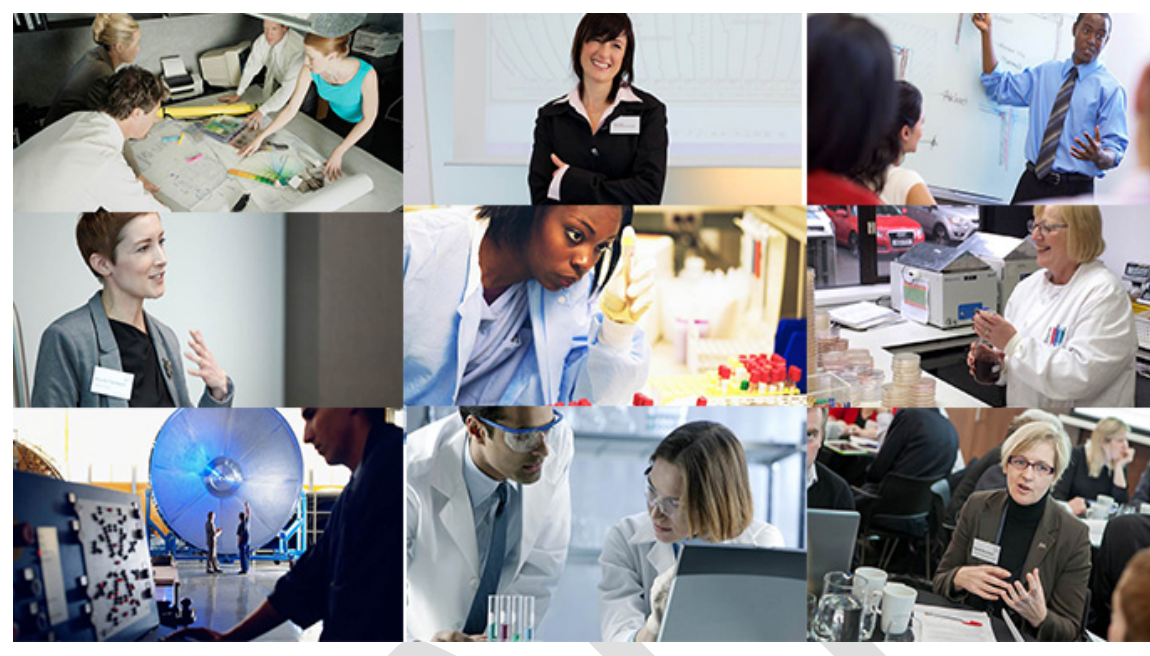

Image Credit: Science Council while genuinely improving and better supporting more stable routes to secure employ where that presently falls short (e.g., in academia). The difficult months of the COVID-19 pandemic, unjust tragic events, protests, and campaigns such as "No Time For Silence", have focused attention on why science and the needs of the world cannot afford for us to be complacent or tardy in making huge strides in diversity and inclusion. Achieving our goals not only needs us to escalate our engagement and sway with a broad sweep of employers, public bodies including funders, and influential figures in STEM and beyond, but also requires us to build understanding and to share this widely. All while coordinating dialogue with other professional societies, trade unions, independent charitable bodies, publishers, policy makers, other stakeholders, and campaign groups. Only by taking this approach can we reach and exceed our ideals of equality, open accessibility (for perspectives refer to Severin et al., 2018; Besançon et al., 2020), and scientific excellence by providing for the varying needs of all through fair equitable practices and the friendliest of workplace environments. What should we be doing and demanding in the face of complex challenges to make sure that these hopes and opportunities lead to our dreams?

\section{Contributions to EAG's blog prepared by the wider geochemical community and their interested partners on topics to constructively inform and help advance all areas relevant to the DEI vision and mission are very welcome and will be gratefully received.}

\section{Expressions of interest can be submitted to dei@eag.eu.com on an ongoing basis.}

The typical length of drafted blogs is roughly one to three A4 pages of an 11 or 12 pt font. Thus, the guidance is to aim for roughly 750 to 2,000 words and to include some images.

Was 2020 the year in which every conceivable thing changed? The recent online screening of "Picture a Scientist" and the provision of a freely available online workshop "Improving Workplace Climate: Responding to Harassment" led by the excellent ADVANCEGeo and hosted by our partner, the Geochemical Society (GS), helped us to recognise many of the types of adverse, unequitable, hostile, and sometimes harmful and traumatic experiences that people can suffer in STEM. Crucially, these informative provisions and the jointly hosted 2020 online GS-EAG Town Halls on "Black Lives Matter" and "Mentoring in Geochemistry" further emphasised the very great importance of:

i) banishing cultures of silence, and fostering kind, equitable, and understanding practices to benefit inclusion and the realisation of peoples' potential;

ii) providing platforms through which the nature and extent of bias is shown to be deeply-embedded in the unconscious of all of us, and;

iii) demonstrating the courage of others in the lengths that some have gone to so as to start the process of making workplace systems fairer and better - these are leaders and allies that we are grateful for. 
This collective work is of long-term impact because it strives to free the system of flawed stereotypical notions, unconscious bias, problematic elitism, cronyism, other unacceptable prejudice, and overt negative conduct associated with inhibiting, demeaning, diverting, forcing or creating fear in others (e.g., unwanted intrusion, practical jokes, gossip, innuendo, gaslighting, blocking of opportunities, assigning menial tasks, overloading, taking or not giving credit for peoples' work and ideas, shouting / swearing / unspoken gestures, or other verbal / physical aggression and abuse linked to harassment, bullying, and / or victimisation). Such contributions, wider published works, and the guidelines of major government and professional bodies prove that we are very far from alone in our convictions to bring about positive professional reforms to improve friendliness while eradicating racism and other bias (see Jermak, 2018; Greider et al., 2019; Agarwal, 2020; Brazil, 2020; Breaking Barriers, Changing Culture, ENAR, Research Integrity, Science Europe, and University Challenge Reports; Cech and Waidzunas, 2021; Devakumar et al., 2020; Dzombak, 2020; EC Diversity and Inclusion Charter; EGU DEI Special Issue, 2020; Fernandes et al., 2020; McKinnon and O'Connell, 2020; Powell et al. 2020; Tager, 2020; van Zyl et al., 2020; and Woolston, 2020 - plus further published examples may be requested of your libraries). In support of the needs of the diversity, equity, and inclusion (DEI) remit in the near term EAG and its community plan to deliver a range of activities such as those pervading the $\mathbf{2 0 2 1}$ Goldschmidt

Conference and a co-sponsored session at EGU. This coming together of values serves to motivate and galvanise us all in the shared efforts required to resolve persistent and system-wide barriers to marginalised people; ensuring that our cultures progress to enable everyone to feel that they belong.

\section{We here define peoples' sense of belonging as feeling happy and comfortable in all relevant settings and professional activities.}

Humans tend to have an innate desire to belong and to feel part of something greater than themselves; the consequences of people not feeling they belong can be severe, and can be linked to the attrition of marginalised groups that is detrimental to the excellence of our scientific community.

To realise positive reforms so that every kind of person can truly thrive the onus for change must be on policies, practices, and majority groups; those being marginalised must be treated equitably, be happy and safe being who they are, and they should not be required to modify themselves or to disguise their identities to conform, fit in, or feel included and secure professionally. After all, it is the stand outs that are to be encouraged, who possess potential to contribute the most innovative, creative and profound of works, and so are to be prized. We must all be self-critical, willing and able to address the sometimes difficult topics and have the hard discussions. Such conversations should not be taken personally but are matters concerning presently deeplyembedded and wide-spread culture, sometimes unintended errors, and are opportunities for each of us to listen actively, and to build comprehension. In doing so the community will gain from sharpened perceptions and understanding of unconscious prejudice, how to ask the 'right' and most incisive questions, as well as how best to be effective allies or correct unintended missteps. This process involves learning from one another the most effective and immediate ways to improve peoples' experiences and build trust while deconstructing barriers through change. Further, the efforts and campaigns to disrupt the status quo, recognise open access publication practices as part of the solution (Irawan et al., 2020; Pourret et al, 2020), and bring additional lasting positive reforms must include buy-in from majority and minoritised people at every level. These actions must not - in themselves - be or become a workload carried almost exclusively, and at potential personal cost, by those talented STEM workers that are being marginalised.

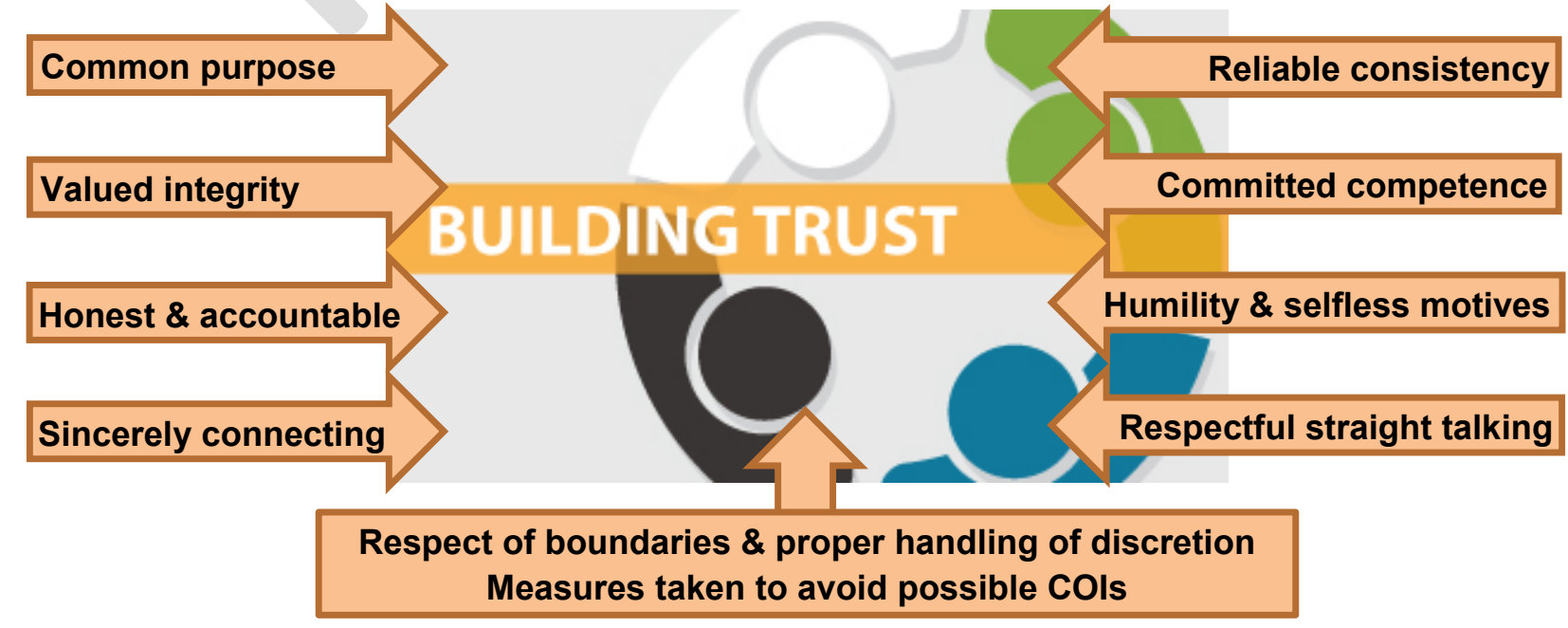


Advancement of DEI in geochemistry and wider STEM communities prompts many questions, both individual and collective. What are you each doing to recognise, access, and fairly share in the work to transform systems and culture alongside the thrill of everyday efforts to reveal and share the rocky, watery, and other mysteries of nature? Is there more that each of you wants to be able to do for and with people of all possible ages, backgrounds, countries, and circumstances? Are any changes needed from managers and among other colleagues to free each and every one of you to achieve these goals in good balance with other professional commitments? Should we, as a community, withhold advancement and recognition from those not proven to give a portion of their time to proactively, positively, and meaningfully participating in the work to make STEM education accessible and inclusive, to advance professional cultures, and to reform the wider STEM working environment? If childcare, shared parental leave, and health care provisions are not available to all staff and students on an equitable and affordable basis, must that be changed? Fieldwork is a key area supporting learning and research in our discipline - be it on land, at sea, or involving studies from flight - and presents several unique challenges to consider with respect to advancing DEI (e.g., Anadu et al., 2020; Demery and Pipkin, 2020; Greene et al., 2020; Demery et al., 2021). How should we acknowledge and give thanks to those selfless individuals who have long campaigned for and devised useful ways to improve community cultures? Where positive and / or affirmative action to accelerate just change is not legally permissible for any sector - academia representing only one small but important part of the whole among which some actionable levers may be entirely lacking - do we have a responsibility as an international society and set of educated individuals to take a position to speak out and to back calls for legislative reform among employment laws? How can we help each other in these endeavours, and how should we garner wider support?

A further matter of particular interest is that in 2020 the Geological Society of America (GSA) announced that alcohol is now prohibited at all poster and oral sessions during its meetings and events. Their announcement provides considered reasons for this change that are sensitive to the differing needs and cultures of all people (among delegates, encompassing students, and potential organisers) thereby demonstrating how this approach is advantageous to inclusion and assured respectful behaviours. Plus, the GSA acknowledges how this decision benefits the image of our professional / learning environments and cultures so as to widen the attractiveness of - at minimum - geochemistry and wider-geoscience. Given this move by GSA related to the society's duty of care for all, what understanding and evidence should we raise to assist EAG leadership in their deliberations on this topic?

Addressing many questions during progress in DEI requires open dialogue and concerted community action. Efforts must be enacted with informed urgency so as to address complex long-standing issues in geochemistry and wider STEM that undermine trust and mutual respect, thereby diminishing the belonging of all people. Trust in the system, in our working culture, and in members of the community requires thought and care to maintain and - even among the more resilient of souls - will require understanding and honest endeavour to rebuild if undermined. Hence, trust must never be taken for granted or its importance underestimated; it is a critical part of the foundations to DEI and requires dedicated effort from all kinds of STEM workers and their administrative support. So too must we dismiss concepts of minoritised people having to be quiet and 'survive' unequal experiences at cost to them and their sense of dignity. Rather, the expected standard should be for fair and friendly working climates that truly listen to and provide for underrepresented people so as to foster equity in support of happy thriving for all. This approach is the only way to ensure that the brightest of global talents are attracted and retained in geochemistry and related areas of STEM so as to reach our as yet unrealised full capacity for excellence.

\section{LESSONS OF COVID-19}

The competitive nature of modern academia and wider STEM employment sectors imparts a broad sweep of demands and expectations on its people at every level. The challenges of such careers have been exacerbated during the difficult times of the COVID-19 pandemic (e.g., Maas et al., 2020). Additional hurdles reflect the many and varied circumstances as well as sometimes abrupt and reactive measures taken to reduce risks for students and all staff in education and research. Critically, helping to protect the loved ones of all - especially those considered to be vulnerable to infection and / or severe COVID-19 symptoms. These efforts have taken place alongside contributions to advance understanding and treatment of the virus - be they intellectual or donations of laboratories, competent staff, or equipment. Thus, events have impacted the community and its members in a variety of ways:

- measure of success under these conditions generally (and should) relate to resilience in keeping going, getting through it together, protecting the vulnerable and supporting those of greater needs, providing for the dignity of 
people who experience personal loss, and being mindful of and kind toward one another more broadly. We have shifted to focusing on our longer-term goals and promise in preference to short-term measures, where the latter can be linked to inequalities in circumstances and access (see also the Declaration on Research Assessment and Viglione, 2020);

- the relationship of the public with key areas of science has changed for the better - including among biomedical themes, tactics to tackle climate change, consciously making choices so as to live more sustainably, and improving environments while safeguarding our planet's natural ecosystems (refer also to progress on the European Green Deal). Plus, we have each been involved in or witnessed the promotion of some major achievements in 2020 international space exploration and planetary science research. These offEarth accomplishments include success in newly collecting extraterrestrial materials from asteroid 101955 Bennu and the nearside of the Moon, plus the return to Earth of material from asteroid 162173 Ryugu, and several missions launched to Mars during 2020's summer. This transformation for the better in science communication concerns both public perspectives and the nature and extent of the provisions that our community and supportive media / publishing outlets make for children and adults to discuss with- and learn from STEM workers of every possible career stage (e.g., Hubenthal et al., 2020). Yet, we are wise to learn from and carefully reflect on the nature of science communication during the unusual and potentially stressful times of the pandemic itself (Tennant et al., 2020).

- varying degrees of educational disruption globally have made remote-learning at home common. In itself this adaptation has demonstrated our flexibility, but has also emphasised the challenges of the digital divide and socioeconomic differences that affect people disproportionately while also threatening to worsen nearterm attainment gaps and stifle the widening of participation during enrolment in higher education;

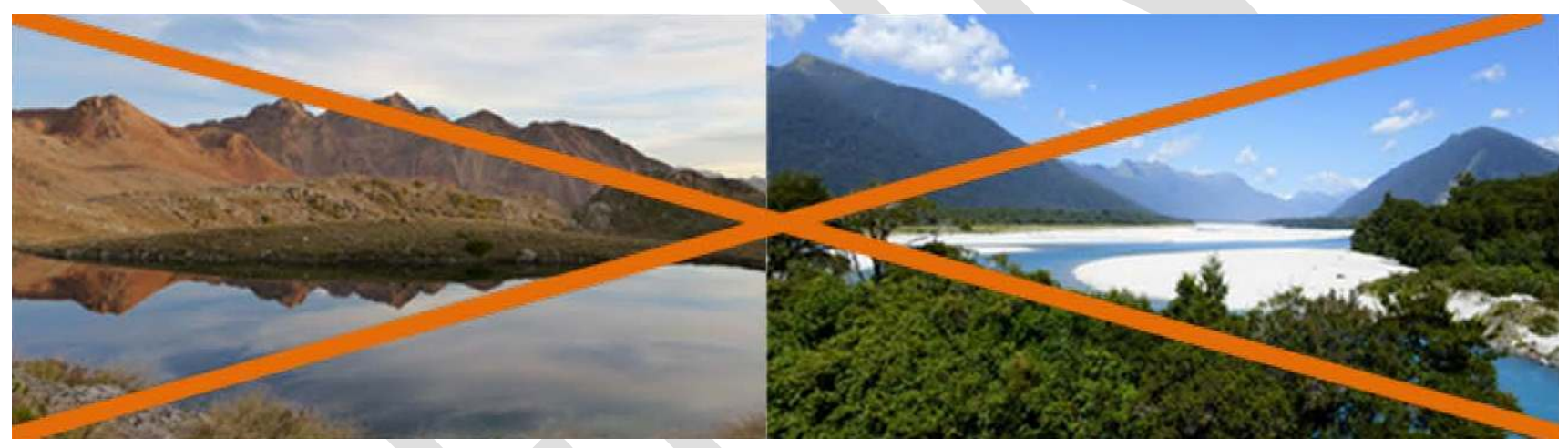

Fieldwork, environmental studies, and other such campaigns have been delayed or cancelled.

- uncertain outlooks have increased, and anxiety / isolation has heightened among students and STEM workers; particular concerns have been raised for the impacts and potential duration of recovery in the Global South (Riedpath and Allotey, 2020). This community concern reflects funding interruptions or contract compromises, shifts in work and travel visa protocols, advice against research visits, museum closures, some redundancies / early-retirements, and cases of department mergers or hiring freezes. Further, cancelled or delayed fieldwork and government geo-/environmental study programs, as well as laboratory shutdowns or repurposing have impacted people. The consequences of all of these matters are changed spheres of reference to be expected among the next generation to be attracted to STEM, and frustration and elevated worries among those of some career areas.

- diversification, fresh opportunities, emergent paradigms, and exciting creative approaches in education, research, and new working areas have opened-up during the past year;

- many people, institutions, and funding bodies have been led to reflect on, provide for, and adjust their working, learning, personalised or group mentoring, and networking styles as well as the working practices available to teams and individuals. This pause for thought is due to multitudes experiencing drawn-out interruptions to their usual efforts or being "safest" or required to work from home (where such buildings, family situations / childcare responsibilities, and outdoor access are hugely variable and some parents have been hit hard; Langin, 2020; Myers et al. 2020). These changes have blurred boundaries that otherwise exist between homes and workplaces;

- awareness has been raised among workplaces, employer and educational leadership, the media, and the general public regarding ongoing challenges and areas of concern with respect to persisting inequalities (including socioeconomic, ethnic, gender identity, sexual orientation, global digital differences, the 
intersections of these and more). These concerns have been given added voice among governments, their advisory groups and policy makers who serve fundamental public interests as well as those of STEM;

- remote access to professional development, conference hosting / attendance, collaborative work across multiple employment sectors, and original ways of building communities, platforming people, and forming new connections has spread while air travel has reduced, and;

- in particular, the extraordinary global accomplishments in addressing the needs and handling of the pandemic itself have made the essential role of well supported, strong, and cooperative national and international scientific bases to significant societal challenges abundantly clear.

Collectively, these impacts have resulted in deep reflection around the globe concerning the overall balance of personal and professional priorities; emphasising our need to think deeply about how best to move forward in solidarity and in new ways that include everyone via the building of trust and increased senses of belonging. Such contemplation has led us to purposefully rethink our personal sense of accomplishment and fulfilment as well as professional measures of success (e.g., Sanders, 2018; Pourret, 2020).

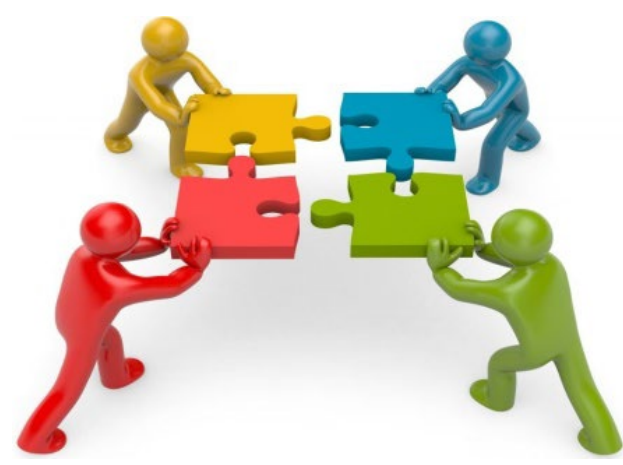

Stronger Together

The times of the COVID-19 pandemic have focused attention on societal and workplace inequalities in new ways while providing for opportunities for the community to speak out and to act with great potential effect. Accelerating lasting change in DEI so that all STEM disciplines better represent the populations that they serve is simply the right and fair thing to do, but should employers or groups require additional incentives there is ample existing evidence proving that competitive advantage is gained because productivity, creativity, and innovation is greatest in diverse and inclusive workplaces (e.g., Nathan and Lee, 2013; Díaz-Garcia et al. 2013; Page, 2017; Hofstra et al., 2020). Data have also long shown that there is a loss of underrepresented people at various career stages due to differences in their professional experiences where they might be or feel disproportionately held back in their work and careers (e.g., Xu, 2008). This attrition and potential 'brain drain' of talented but minoritised people to alternative destinations is deleterious for employers and staff groups, but can be abated if workplace environments enhance friendly belonging, are effective in emboldening every kind of person, and achieve equity in provisions and employment circumstances at every level. Improving retention of talented people has the consequence of reducing potential disruption, possible negative impacts on workplace morale, additional work and costs associated with personnel changes. Further, generational differences in values and workforce demographics mean that diversity and inclusion among colleagues and leadership is increasingly important to the reputations and attractiveness of a given body on national and global stages. Hence, credible commitment to- and proven progress in all areas of DEI benefits recruitment as well as retention of talent. Funders (and customers) of employers can and should support the work involved while bolstering incentives to improve DEI, and these entities can play a key role in monitoring and holding organisations to account.

For the variety of reasons explored above part of the EAG DEI Committee's work over the coming months has the aim of acquiring knowledge to advance our understanding of the current status of the geochemistry and cosmochemistry community so as to provide a benchmark, share such new information, and plan for our progress. This approach necessitates two-way dialogue to gain a better understanding of the current make-up of our people and the condition of our culture, as well as learning from successful examples of approaches to reform. In this respect we are excited to encourage your contributions to the variety of EAG-GS DEI-facing activities, social media campaigns, and to this society blog.

In conclusion, we hope that - while courteously and empathetically supporting one another at these unprecedented times - every person is and will resolve to direct a fair share of their energies to participate in all forms of constructive efforts that scrutinise and improve community cultures, workplace practices, related policies, structures, and legislative frameworks. We very much look forward to effective teamwork with you all!

\section{ACKNOWLEDGEMENTS}

This contribution was informed by discussions and electronic exchanges with the entire membership of European Association of Geochemistry's Diversity, Equity, and Inclusion Committee as well as the equivalent Committee of the Geochemical Society. Our EAG DEI Committee is here to help support the community, listen to and learn from your comments should you wish to contact us - dei@eag.eu.com. 


\section{REFERENCES}

Agarwal, P., 2020. Sway: Unravelling Unconscious Bias. Bloomsbury Sigma [Book]

Anadu, J., H. Ali, and C. Jackson (2020), Ten steps to protect BIPOC scholars in the field, Eos, 101, https://doi.org/10.1029/2020EO150525

Besançon, L., Peiffer-Smadja, N., Segalas, C., Jiang, H., Masuzzo, P., Smout, C.A., Billy, E., Deforet, M. and Leyrat, C., 2020. Open science saves lives: Lessons from the COVID-19 pandemic. BioRxiv, Link

Brazil, R., 2020. Gold standard or below par? Chemistry World, Careers. Link

Cech E. A., and Waidzunas T. J., 2021. Systemic inequalities for LGBTQ professionals in STEM. Science Advances, 7(3), eabe0933 https://advances.sciencemag.org/content/7/3/eabe0933

Demery, A.J. and Pipkin, M., 2020. Safe Fieldwork Strategies for At-Risk Individuals. Open Access Link

Demery, A.J.C. and Pipkin, M.A., 2021. Safe fieldwork strategies for at-risk individuals, their supervisors and institutions. Nature Ecology \& Evolution, 5(1), pp.5-9. Open Access Link

Devakumar, D., Bhopal, S.S. and Shannon, G., 2020. COVID-19: the great unequaliser. Journal of the Royal Society of Medicine, 113(6), pp.234-235. Open Access Link

Díaz-García, C., González-Moreno, A. and Jose Saez-Martinez, F., 2013. Gender diversity within R\&D teams: Its impact on radicalness of innovation. Innovation, 15(2), pp.149-160. www.tandfonline.com/doi/abs/10.5172/impp.2013.15.2.149

Dzombak, R., 2020. Queer visibility in geoscience has been almost non-existent for decades. A new generation is working to change that. GSA, Speaking of Geoscience. Open Access Link

Fernandes, A.M., Abeyta, A., Mahon, R.C., Martindale, R., Bergmann, K.D., Jackson, C.A.L., Present, T.M., Reano, D., Swanson, T. and Butler, K., 2020. "Enriching Lives within Sedimentary Geology": Actionable Recommendations for Making SEPM a Diverse, Equitable and Inclusive Society for All Sedimentary Geologists. The Sedimentary Record, www.sepm.org/files/183article.ly3dwq3vr5refg6g.pdf

Greene, S., Ashley, K., Dunne, E., Edgar, K., Giles, S. and Hanson, E., 2020. Toilet stops in the field: An educational primer and recommended best practices for field-based teaching. https://osf.io/gnhj2/

Greider, C.W., Sheltzer, J.M., Cantalupo, N.C., Copeland, W.B., Dasgupta, N., Hopkins, N., Jansen, J.M., Joshua-Tor, L., McDowell, G.S., Metcalf, J.L. and McLaughlin, B., 2019. Increasing gender diversity in the STEM research workforce. Science, 366(6466), pp.692-695. Link

Hofstra, B., Kulkarni, V.V., Galvez, S.M.N., He, B., Jurafsky, D. and McFarland, D.A., 2020. The Diversity-Innovation Paradox in Science. Proceedings of the National Academy of Sciences, 117(17), pp.9284-9291. Open Access Link

Hubenthal, M., Bohon, W., and Taber, J., 2020. A pandemic pivot in Earth science outreach and education, Eos, 101, https://doi.org/10.1029/2020EO152146.

Irawan, D.E., Hedding, D., and Pourret, O., 2020. How Open Science may help us during and after the pandemic. EGU Blog, Open Access Link

Jermak, H.E., Lucatello, S. and Woods, P., 2018. Equity and diversity in astronomy. Nature Astronomy, 2; 523-524. https://doi.org/10.1038/s41550-018-0514-0

Langin, K., 2020. Pandemic hits scientist parents hard. Science, Careers. Open Access Link

Maas, B., Grogan, K.E., Chirango, Y., Harris, N., Liévano-Latorre, L.F., McGuire, K.L., Moore, A.C., Ocampo-Ariza, C., Palta, M.M., Perfecto, I. and Primack, R.B., 2020. Academic leaders must support inclusive scientific communities during COVID-19. Nature Ecology \& Evolution, pp.1-2. Open Access. www.nature.com/articles/s41559-020-1233-3

McKinnon, M. and O'Connell, C., 2020. Perceptions of stereotypes applied to women who publicly communicate their STEM work. Humanities and Social Sciences Communications, 7(1), pp.1-8. Open Access Link

Myers, K.R., Tham, W.Y., Yin, Y., Cohodes, N., Thursby, J.G., Thursby, M.C., Schiffer, P., Walsh, J.T., Lakhani, K.R. and Wang, D., 2020. Unequal effects of the COVID-19 pandemic on scientists. Nature Human Behaviour, 4(9), pp.880-883. Open Access Link

Nathan, M. and Lee, N., 2013. Cultural Diversity, Innovation, and Entrepreneurship: Firm-level Evidence from London. Economic Geography, 89(4), pp.367-394. https://onlinelibrary.wiley.com/doi/pdf/10.1111/ecge.12016

Page, S.E., 2019. The diversity bonus: How great teams pay off in the knowledge economy. Princeton University Press [Book].

Pourret, O., 2020. Redefine Success After Lock Downs. Nature, 583, pp. 646. Open Access Link

Pourret, O., Irawan, D.E., Tennant, J.P., Hursthouse, A. and van Hullebusch, E.D., 2020. The growth of open access publishing in geochemistry. Results in Geochemistry, https://doi.org/10.1016/j.ringeo.2020.100001

Powell, K., Terry, R. and Chen, S., 2020. How LGBT+ scientists would like to be included and welcomed in STEM workplaces. Nature, 586(7831), pp.813-816. Open Access www.nature.com/articles/d41586-020-02949-3

Reidpath, D. and Allotey, P., 2020. Preserve Global South's research capacity. Science, 368(6492), pp.725-725. https://science.sciencemag.org/content/368/6492/725

Sanders, J., 2018. Why we must redefine success. Chemistry World, Opinion. Link

Severin, A., Egger, M., Eve, M.P. and Hürlimann, D., 2018. Discipline-specific open access publishing practices and barriers to change: an evidence-based review. F1000Research, 7. Open Access Link

Tager, R., 2020. Royal Society of Chemistry publishes first analysis of diversity. Chemistry World, News. Link promoting the publication of the RSC Diversity Data Report 
Tennant, J. P., Illingworth, S., Stewart, I., and von Elverfeldt, K.: Editorial: Geoscience in a time of pandemics, Geoscience Communication, 3, 71-72, https://doi.org/10.5194/gc-3-71-2020

van Zyl, M., Haynes, E.M., Batchelar, D. and Jakobi, J.M., 2020. Examining gender diversity growth as a model for inclusion of all underrepresented persons in medical physics. Medical Physics. Open Access Link

Viglione, G., 2020. Are women publishing less during the pandemic? Here's what the data say. Nature, 581(7809), pp.365366. www.nature.com/articles/d41586-020-01294-9

Woolston, C., 2020. It's like we're going back 30 years': how the coronavirus is gutting diversity in science. Nature, Career Feature. Open Access Link

Xu, Y.J. Gender Disparity in STEM Disciplines: A Study of Faculty Attrition and Turnover Intentions. Res High Educ 49, 607-624 (2008).https://doi.org/10.1007/s11162-008-9097-4

\section{DRAWING SPECIAL ATTENTION TO CROSS-CUTTING MATERIALS AND IDEAS:}

Breaking Barriers Reports. Among which are the 2018-2019 and 2019-2020 reports.

Changing Culture Report. Open Access Link, related new guidelines, and 2-years on report.

Declaration on Research Assessment (DORA). https://sfdora.org/ Are you and / or your employer DORA signatories? See also the DORA brief on unintended cognitive and systems bias ( $\underline{\text { link }})$ and the DORA blog series $(\underline{\text { link }})$.

EGU DEI Special Issue, 2020, Advances in Geoscience, 53. Open Access Link

European Commission, Diversity and Inclusion Charter. Open Access Link, further information on signatories, and statement pertaining to ERC. Plus, European Charter for Researchers - Open Access Link.

European Network Against Racism, Vision and mission and Theory of Change 2018-2021

European Trade Union Committee for Education (ETUCE), link and their equality focus (link).

Research Integrity: A Landscape Study. Open Access Link1, Link2 and broadly related Researcher Development Concordat.

Science Europe, Gender and Diversity. Link to position statement and resources.

University Challenge Report. Open Access Link and Link2 to other student minds reports.

\section{Author Bios}

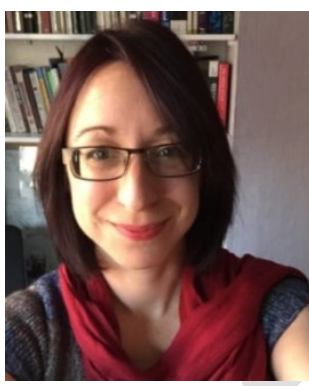

Dr. Amy J. V. Riches served as lead author of this contribution and is a Founding Member and Co-Chair of EAG's Diversity, Equity, and Inclusion Committee. She is currently revelling Freelance as a writer / editor, is a Visiting Honorary Fellow at the University of Edinburgh, UK, and a SETI Affiliate Scientist, Carl Sagan Centre, USA. Amy is committed to improving community cultures to advance DEI. She intends for this work to benefit science for good and to assist all people to happily fulfil their potentials while inspiring subsequent generations.

a,PlanetaryAmy

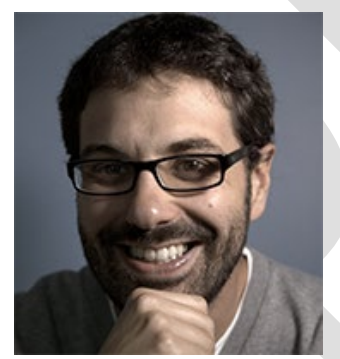

Olivier Pourett is an Associate Professor of Geochemistry at Université of LaSalle, France. He is among the Founding Members of EAG's DEI Committee, coordinates the joint GS-EAG DEI Writing Task Force, spearheads advocacy for Open Science, just Science done right, and campaigns for disability and caring awareness. @,olivier pourret

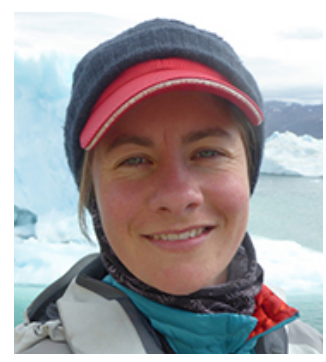

Susan Little is a Lecturer and NERC Independent Research Fellow at University College London, UK. She is also an EAG Councillor and Co-Chair of the EAG Diversity, Equity and Inclusion Committee. Susan aims to help the society and motivate the community to accelerate its efforts to improve the representation of diverse groups within the geochemical community.

a,suselittle

To remain aware and join in with a range of activities that help the community to advance the DEI remit do please contribute to, look out for, discuss and share the regular features in EAG newsletters and social media updates.

y The European Association of Geochemistry can be followed at @EAG_and an EAG twitter account dedicated to promoting all members of the community while advocating for DEI is expected to launch during February 2021. 